\title{
A descoberta das correntes neutras das interações fracas
}

\author{
E.C.F.S. Fortes ${ }^{1}$, M.C. Tijero e V. Pleitez
}

Na p. 422, na legenda da Fig. 5 afirma-se que esta corresponde à "Fotografia do evento de um elétron na GARGAMELLE" . Na verdade, essa figura corresponde ao processo hadrônico $\nu_{\mu}+p \rightarrow \nu_{\mu}+p+\pi^{0}$. A fonte dessa figura é G.D. Coughlan, J.E. Dodd, e B.M. Gripaios, The Ideas of Particle Physics (Cambridge University Press, Cambridge, 1991), $3^{\text {a }}$ ed., p. 113. Uma figura desse tipo de evento aparece pela primeira vez em F.J. Hasert et al., Observation of Neutrino-like Interactions without Muon or Electron in the Gargamelle Neutrino Experiment, Nucl. Phys. B73, 1 (1974), mas a sua resolução não é boa e por isso não foi reproduzida aqui.

A figura do evento de um elétron é a que aparece aqui. Fonte: F.J. Hasert et al., Search for Elastic Muon-Neutrino Electron Scattering, Phys. Lett. B46, 121 (1973). A legenda: "Possível evento do tipo $\bar{\nu}_{\mu}+e^{-} \rightarrow \bar{\nu}_{\mu}+e^{-"}$ corresponde à legenda do artigo original.

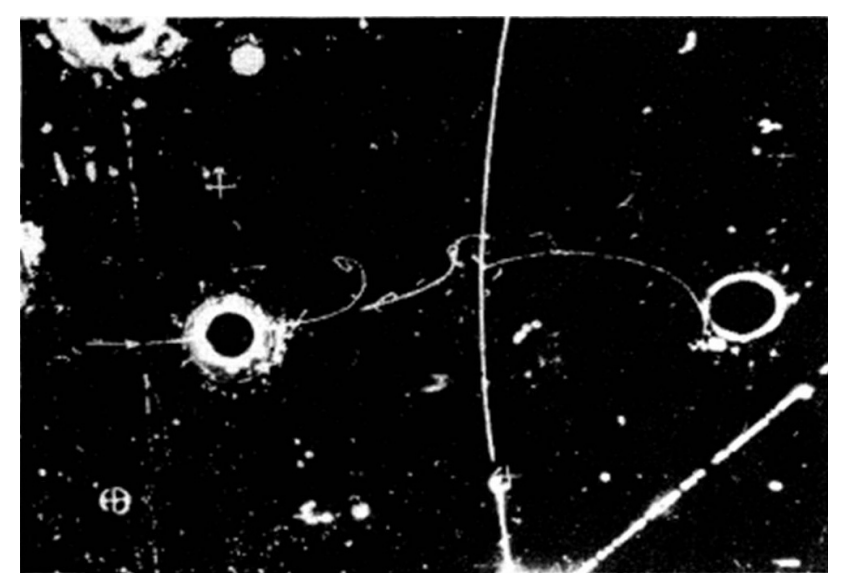

Figura 1 - Possível evento do tipo $\bar{\nu}_{\mu}+e^{-} \rightarrow \bar{\nu}_{\mu}+e^{-}$.

Revista Brasileira de Ensino de Física, v. 30, n. 1, 1902 (2008) www.sbfisica.org.br

Erratum RBEF 29, 645 (2007)

\section{Exact solution for the nonlinear pendulum}

\section{A. Beléndez², C. Pascual, D. I. Méndez, T. Beléndez and C. Neipp}

Equation (32) should be replaced by the following equation

$$
\theta(t)=\theta_{0} \cos \left[\omega\left(\theta_{0}\right) t\right]
$$

\footnotetext{
${ }^{1}$ E-mail: vicente@ift.unesp.br.

${ }^{2}$ E-mail: a.belendez@ua.es. 